\title{
WEAK-TYPE WEIGHTS AND NORMABLE LORENTZ SPACES
}

\author{
MARÍA J. CARRO, ALEJANDRO GARCÍA DEL AMO, AND JAVIER SORIA
}

(Communicated by J. Marshall Ash)

\begin{abstract}
We show that the Lorentz space $\Lambda^{1}(w)$ is a Banach space if and only if the Hardy-Littlewood maximal operator $M$ satisfies a certain weak-type estimate. We also consider the case of general measures. Finally, we study some properties of several indices associated to these spaces.
\end{abstract}

\section{INTRODUCTION}

We are going to study weighted Lorentz spaces of functions defined in $\mathbb{R}^{n}$ as follows (for standard notation we refer to [BS] and [GR]): If $u$ is a weight in $\mathbb{R}^{n}$, $w$ is a weight in $\mathbb{R}^{+}, f_{u}^{*}$ denotes the decreasing rearrangement of $f$ with respect to the measure $u(x) d x$ and $0<p<\infty$, we define

$$
\Lambda_{u}^{p}(w)=\left\{f ;\|f\|_{\Lambda_{u}^{p}(w)}=\left(\int_{0}^{\infty}\left(f_{u}^{*}(t)\right)^{p} w(t) d t\right)^{1 / p}<\infty\right\} .
$$

If $u \equiv 1$, we will only write $\Lambda^{p}(w)$. Classical examples are obtained by choosing $w(t)=t^{(p / q)-1}$. In this case $\Lambda^{p}(w)=L^{q, p}$. A classical result of G.G. Lorentz (see [Lo]) shows that $\|\cdot\|_{\Lambda^{1}(w)}$ is a norm, if and only if, $w$ is a decreasing function. The problem of finding conditions on $w$ so that $\Lambda^{p}(w)$ is a Banach space (that is, there exists a norm equivalent to $\|\cdot\|_{\Lambda^{p}(w)}$ ) was solved, for $p>1$, by E. Sawyer ([Sa]). This condition is that the Hardy-Littlewood maximal operator is bounded on $\Lambda^{p}(w)$. The weights for which this holds were first characterized by M.A. Ariño and B. Muckenhoupt $([\mathrm{AM}])$, and it is known as the $B_{p}$ condition: there exists $C>0$ such that, for all $r>0$,

$$
r^{p} \int_{r}^{\infty} \frac{w(x)}{x^{p}} d x \leq C \int_{0}^{r} w(x) d x .
$$

It is clear that (1) is not the right condition for $p=1$, since with $w \equiv 1$, we have that $\Lambda^{1}(w)=L^{1}$ is a Banach space, but $w$ does not satisfy (1). It is well known that the weighted strong-type and weak-type boundedness of the Hardy-Littlewood maximal operator coincide for $p>1$. This motivates to consider the same kind of weak-type estimates for the spaces $\Lambda_{u}^{p}(w)$. To this end we recall the following definition:

Received by the editors September 14, 1994.

1991 Mathematics Subject Classification. Primary 42B25, 46E30.

The first and third authors were partially supported by DGICYT PB94-0879.

The second author was partially supported by DGICYT PB94-0243. 
Definition 1.1 ([CS1]). Let $u$ and $w$ be weights as above and $0<p<\infty$. We define

$$
\Lambda_{u}^{p, \infty}(w)=\left\{f ;\|f\|_{\Lambda_{u}^{p, \infty}(w)}=\sup _{y>0} y\left(\int_{0}^{\lambda_{f}^{u}(y)} w(t) d t\right)^{1 / p}<\infty\right\},
$$

where $\lambda_{f}^{u}$ is the distribution function of $f$ with respect to the measure $u(x) d x$.

Observe that $\|f\|_{\Lambda_{u}^{p, \infty}(w)}=\sup _{x>0} f_{u}^{*}(x)\left(\int_{0}^{x} w(t) d t\right)^{1 / p}$. Also, for the weight $w(t)=t^{(p / q)-1}$, we have that $\Lambda_{u}^{p, \infty}(w)=L_{u}^{q, \infty}$. Our main result is the fact that in order to fully characterize when $\Lambda^{p}(w)$ is a Banach space, for the whole range $1 \leq p<\infty$, we should replace the boundedness of $M$ in $\Lambda^{p}(w)$ (as in [Sa]) by the a priori weaker condition on the maximal operator $M: \Lambda^{p}(w) \longrightarrow \Lambda^{p, \infty}(w)$. We give the details in section 2 . In section 3 we study the case of a general measure and in section 4 we define an index associated to $\Lambda^{p}(w)$ and show several characterizations of this index in terms of the $B_{p}$ condition.

\section{WEAK-TYPE WEIGHTS}

Let us recall the following characterization of the weak-type boundedness of $M$ for $p>1$ (see Theorem 3.9 in [CS3]):

Theorem 2.1. If $p>1$, then $M: \Lambda^{p}(w) \longrightarrow \Lambda^{p, \infty}(w)$, if and only if, there exists $C>0$ such that for all $r>0$

$$
\left(\int_{0}^{r}\left(\frac{1}{x} \int_{o}^{x} w(t) d t\right)^{-p^{\prime}} w(x) d x\right)^{1 / p^{\prime}}\left(\int_{0}^{r} w(t) d t\right)^{1 / p} \leq C r
$$

It is easy to show that (2) is equivalent to (1) (see [Sa]) and hence, it is also equivalent to the strong-type boundedness $M: \Lambda^{p}(w) \longrightarrow \Lambda^{p}(w)$. The case $p=1$ of (2) motivates the following definition:

Definition 2.2. We say that $w \in B_{1, \infty}$ if there exists $C>0$ such that for all $0<s \leq r<\infty$

$$
\frac{1}{r} \int_{0}^{r} w(t) d t \leq \frac{C}{s} \int_{0}^{s} w(t) d t
$$

If we set $W(x)=\int_{0}^{x} w(t) d t$, then $w \in B_{1, \infty}$ is equivalent to saying that $W$ is quasi-concave (see [KPS]). We now prove our main theorem (which is the weak-type version of the main theorem in $[\mathrm{AM}]$ and the end point case $p=1$ of [Sa]). Recall that $S f(x)=x^{-1} \int_{0}^{x} f(t) d t$ is the Hardy operator, $f^{* *}(t)=S f^{*}(t)$ and $L_{\mathrm{dec}}^{1}(w)$ is the set of all positive decreasing functions in $L^{1}(w)$.

Theorem 2.3. The following conditions are equivalent:

(i) $\Lambda^{1}(w)$ is a Banach space.

(ii) $w \in B_{1, \infty}$.

(iii) $M: \Lambda^{1}(w) \longrightarrow \Lambda^{1, \infty}(w)$ is bounded.

(iv) For all $r>0,\left\|r t^{-1} \chi_{\{t \geq r\}}\right\|_{L^{1, \infty}(w)} \leq C\left\|\chi_{(0, r)}\right\|_{L^{1}(w)}$.

(v) $S: L_{\mathrm{dec}}^{1}(w) \longrightarrow L^{1, \infty}(w)$. 
Proof. Let us first show the equivalence of (ii), (iii) and (v). By definition, $M$ : $\Lambda^{1}(w) \longrightarrow \Lambda^{1, \infty}(w)$ is bounded, if and only if

$$
\sup _{y>0} y \int_{0}^{\lambda_{M f}(y)} w(t) d t \leq C\|f\|_{\Lambda^{1}(w)}=C\left\|f^{*}\right\|_{L^{1}(w)} .
$$

Now since $(M f)^{*} \approx f^{* *}$ and $\lambda_{M f}=\lambda_{(M f)^{*}}(y)$ (see [BS]), we obtain that (3) is equivalent to

$$
\sup _{y>0} y \int_{0}^{\lambda_{S f}(y)} w(t) d t \leq C\|f\|_{L^{1}(w)}
$$

for all $f \in L_{\mathrm{dec}}^{1}(w)$. Therefore (4) is the same as saying that

$$
S: L_{\mathrm{dec}}^{1}(w) \longrightarrow \Lambda^{1, \infty}(w) .
$$

Now it is easy to observe that if $f$ is a decreasing function, then $\|S f\|_{\Lambda^{1, \infty}(w)}=$ $\|S f\|_{L^{1, \infty}(w)}$ (which proves (v)). But Theorem 3.3-(b) in [CS2] shows that this is equivalent to $w \in B_{1, \infty}\left(\right.$ take $w_{0}=w_{1}=w, p_{0}=p_{1}=1$ and $k(x, t)=x^{-1} \chi_{(0, x)}(t)$ in that theorem). Assume now (i) holds and let us show (ii). If $\Lambda^{1}(w)$ is a Banach space, then there exists an equivalent norm on $\Lambda^{1}(w),\|\cdot\| \approx\|\cdot\|_{\Lambda^{1}(w)}$ and hence, there exists $C>0$ such that for all $N \in \mathbb{N}$ and $g_{1}, \cdots, g_{N} \in \Lambda^{1}(w), \| g_{1}+\cdots+$ $g_{N} \|_{\Lambda^{1}(w)} \leq C\left(\left\|g_{1}\right\|_{\Lambda^{1}(w)}+\cdots+\left\|g_{N}\right\|_{\Lambda^{1}(w)}\right)$. Suppose first $r=2^{k} s$, with $k=1,2, \cdots$. Set $f=\chi_{\left(0,2^{k} s\right)}$ and $f_{j}=\chi_{(j s,(j+1) s)}, j=0,1, \cdots, 2^{k}-1$. Let $F$ and $F_{j}$ be functions defined in $\mathbb{R}^{n}$ such that $F^{*}=f, F_{j}^{*}=f_{j}^{*}=\chi_{(0, s)}$ and $F=\sum_{j} F_{j}$. Now,

$$
W\left(2^{k} s\right)=\|F\|_{\Lambda^{1}(w)} \leq C \sum_{j=0}^{2^{k}-1}\left\|F_{j}\right\|_{\Lambda^{1}(w)}=C 2^{k} W(s) .
$$

Finally, for a general $r>s$, let $k=1,2, \cdots$ be such that $2^{k-1} s \leq r<2^{k} s$. Then

$$
\frac{1}{r} \int_{0}^{r} w(t) d t \leq \frac{1}{2^{k-1} s} W\left(2^{k} s\right) \leq \frac{C 2^{k}}{2^{k-1} s} W(s)=\frac{2 C}{s} \int_{0}^{s} w(t) d t .
$$

To see the converse, we use Theorem 1.1 of [KPS, $\S \mathrm{II}]$ and obtain that there exists a decreasing weight $v$ so that if $V(x)=\int_{0}^{x} v(t) d t$, then $W \approx V$, and it is now easy to show that $\Lambda^{1}(v)$ is a Banach space and $\Lambda^{1}(w)=\Lambda^{1}(v)$. To finish, we observe that

$$
\left\|r t^{-1} \chi_{\{t \geq r\}}\right\|_{L^{1, \infty}(w)}=\sup _{y \leq 1} y \int_{r}^{r / y} w(t) d t
$$

and hence, (iv) is equivalent to

$$
\sup _{y \leq 1} \frac{y}{r} \int_{0}^{r / y} w(t) d t \leq C \frac{1}{r} \int_{0}^{r} w(t) d t
$$

which is equivalent to (ii). 
Remarks 2.4. (a) The equivalence between (i) and (ii) was already known, as a consequence of the study of the $p$-convexity of a rearrangement invariant space (we want to thank Professor Y. Raynaud for pointing out this to us).

(b) It is now clear that, by (iii), $B_{1} \subset B_{1, \infty}$. It is also true that $B_{1, \infty} \subset B_{p}$, for $p>1$. In fact, if $r>0$, we have just shown that for all $k=1,2, \cdots, W\left(2^{k} r\right) \leq$ $C 2^{k} W(r)$, and hence,

$$
\begin{aligned}
\int_{r}^{\infty} \frac{w(t)}{t^{p}} d t & =\sum_{k=0}^{\infty} \int_{2^{k} r}^{2^{k+1} r} \frac{w(t)}{t^{p}} d t \leq \frac{1}{r^{p}} \sum_{k=0}^{\infty} \frac{1}{2^{k p}} \int_{0}^{2^{k+1} r} w(t) d t \\
& \leq \frac{1}{r^{p}} \sum_{k=0}^{\infty} \frac{C 2^{k+1}}{2^{k p}} \int_{0}^{r} w(t) d t \leq \frac{C}{r^{p}}\left(\sum_{k=0}^{\infty} \frac{1}{2^{k(p-1)}}\right) \int_{0}^{r} w(t) d t
\end{aligned}
$$

(c) If we set $\|f\|_{*}=\sup _{y>0} f^{* *}(y) W(y)$, then $\|\cdot\|_{*}$ is a norm, and if $w \in$ $B_{1, \infty}$, then $\|f\|_{*} \leq C\|f\|_{\Lambda^{1}(w)}$. Sometimes the converse inequality is also true, and hence $\|\cdot\|_{*}$ is the equivalent norm in $\Lambda^{1}(w)$. This happens, for example, if $w \equiv 1$ (see $[\mathrm{Sj}]$ for related results). The equivalence is not true in general: if $w(t)=t^{-1 / 2}$, then $\Lambda^{1}(w)$ is a Banach space, but if $f^{*}(t)=t^{-1 / 2}$, then $f \notin \Lambda^{1}(w)$ and $\|f\|_{*}<\infty$. However, if $y^{-1} W(y)$ is equivalent to an increasing function, then $\|f\|_{\Lambda^{1}(w)} \leq C\|f\|_{*}$. It is also easy to show that this condition is not necessary.

(d) For some related results see $[\mathrm{Ne}, \S 6]$.

\section{Extensions to General measures}

We want to study geometric properties of the spaces $\Lambda_{u}^{p}(w)$, for general weights. For example, it was proved in [CS1] that $\|\cdot\|_{\Lambda_{u}^{p}(w)}$ is a quasinorm, if and only if $W$ satisfies a certain doubling condition $\left(W \in \Delta_{2}\right)$. Also it is an easy exercise to show that these spaces are always complete (as long as $W(x)>0$ if $x>0$ ). We now ask the same question we did for $\Lambda^{p}(w)$ : under which conditions are they Banach spaces? We observe that Theorem 2.3 does not hold in general, since for $w \equiv 1$, $\Lambda_{u}^{p}(w)=L_{u}^{p}$, which is a Banach space if $p \geq 1$, but the Hardy-Littlewood maximal operator is not always bounded $M: L_{u}^{p} \longrightarrow L_{u}^{p, \infty}$. However, we can show that, in many cases, this property only depends on the weight $w$ :

Theorem 3.1. If $u \notin L^{1}$ (or $d u$ is an infinite non-atomic measure) and $p>0$, then $\Lambda_{u}^{p}(w)$ is a Banach space, if and only if $\Lambda^{p}(w)$ is a Banach space.

Proof. By a symmetric argument, it suffices to consider the case $\Lambda^{p}(w)$ Banach: we want to show that there exists $C>0$ so that for simple functions $S_{1}, \cdots, S_{N}$,

$$
\left\|\sum_{j=1}^{N} S_{j}\right\|_{\Lambda_{u}^{p}(w)} \leq C \sum_{j=1}^{N}\left\|S_{j}\right\|_{\Lambda_{u}^{p}(w)},
$$

since the Monotone Convergence Theorem implies (5) for arbitrary functions, and it is easy to show that this implies the existence of an equivalent norm. We will only consider the case $N=2$ (the case $N>2$ is completely analogous). So, let $S_{j}=\sum_{k} \lambda_{k}^{j} \chi_{A_{k}^{j}}, j=1,2$, and the sets $A_{k}^{j}$ are pairwise disjoint. Then,

$$
S_{1}+S_{2}=\sum_{k} \lambda_{k}^{1} \chi_{A_{k}^{1} \backslash \cup_{j} A_{j}^{2}}+\sum_{k} \lambda_{k}^{2} \chi_{A_{k}^{2} \backslash \bigcup_{j} A_{j}^{1}}+\sum_{k, j}\left(\lambda_{k}^{1}+\lambda_{j}^{2}\right) \chi_{A_{k}^{1} \cap A_{j}^{2}} .
$$


We choose a family of disjoint sets $\left\{E_{k}^{1}, E_{j}^{2}, I_{k, j}: k, j\right\}$ such that

$$
\begin{aligned}
& \left|E_{k}^{1}\right|=u\left(A_{k}^{1} \backslash \bigcup_{j} A_{j}^{2}\right), \\
& \left|E_{k}^{2}\right|=u\left(A_{k}^{2} \backslash \bigcup_{j} A_{j}^{1}\right), \\
& \left|I_{k, j}\right|=u\left(A_{k}^{1} \cap A_{j}^{2}\right),
\end{aligned}
$$

where, as usual, for a set $A \subset \mathbb{R}^{n}, u(A)=\int_{A} u(x) d x$. Define also,

$$
\begin{aligned}
C_{1} & =\sum_{k} \lambda_{k}^{1} \chi_{E_{k}^{1} \cup\left(\cup_{j} I_{k, j}\right)}, \\
C_{2} & =\sum_{k} \lambda_{k}^{2} \chi_{E_{k}^{2} \cup\left(\cup_{j} I_{j, k}\right)},
\end{aligned}
$$

so that $C_{1}^{*}=\left(S_{1}\right)_{u}^{*}, C_{2}^{*}=\left(S_{2}\right)_{u}^{*}$ and $\left(C_{1}+C_{2}\right)^{*}=\left(S_{1}+S_{2}\right)_{u}^{*}$. Finally,

$$
\begin{aligned}
\left\|S_{1}+S_{2}\right\|_{\Lambda_{u}^{p}(w)} & =\left\|C_{1}+C_{2}\right\|_{\Lambda^{p}(w)} \leq C\left(\left\|C_{1}\right\|_{\Lambda^{p}(w)}+\left\|C_{2}\right\|_{\Lambda^{p}(w)}\right) \\
& =C\left(\left\|S_{1}\right\|_{\Lambda_{u}^{p}(w)}+\left\|S_{2}\right\|_{\Lambda_{u}^{p}(w)}\right) \cdot \square
\end{aligned}
$$

This result allows us to extend $(2.6)$ of $[\mathrm{Hu}]$ :

Corollary 3.2. Let $u \notin L^{1}$ (or $d u$ an infinite non-atomic measure) let $p>0$, and suppose $\Lambda_{u}^{p}(w)$ is a Banach space. Then $p \geq 1$.

Proof. From the previous theorem we see that it is enough to consider $u \equiv 1$. Assume now that $0<p<1$. As in $[\mathrm{Hu}]$, we are going to find a sequence of functions $\left\{f_{j}\right\}_{j}$, satisfying

$$
\left\|f_{j}\right\|_{\Lambda^{p}(w)}=1 \quad \text { but } \quad \frac{1}{N}\left\|\sum_{k=1}^{N} f_{k}\right\|_{\Lambda^{p}(w)} \underset{N \rightarrow \infty}{\longrightarrow} \infty
$$

Choose $A_{k} \subset \mathbb{R}^{n}, \cdots \subset A_{k+1} \subset A_{k}$, such that $W\left(\left|A_{k}\right|\right)=2^{-p k}$. Let $f_{k}=2^{k} \chi_{A_{k}}$. Then,

$$
\left\|f_{k}\right\|_{\Lambda^{p}(w)}=2^{k}\left(\int_{0}^{\infty}\left(\left(\chi_{A_{k}}\right)^{*}\right)^{p} w(t) d t\right)^{1 / p}=2^{k} W\left(\left|A_{k}\right|\right)^{1 / p}=1
$$


But, if for $N$ fixed, we set $A_{N+1}=\emptyset$, then,

$$
\begin{aligned}
\frac{1}{N}\left\|\sum_{k=1}^{N} f_{k}\right\|_{\Lambda^{p}(w)} & =\frac{1}{N}\left\|\sum_{k=1}^{N}\left(\sum_{j=1}^{k} 2^{j}\right) \chi_{\left(A_{k} \backslash A_{k+1}\right)}\right\|_{\Lambda^{p}(w)} \\
& =\frac{1}{N}\left[\int_{0}^{\infty}\left(\left[\sum_{k=1}^{N}\left(\sum_{j=1}^{k} 2^{j}\right) \chi_{\left(A_{k} \backslash A_{k+1}\right)}\right]^{*}\right)^{p} w(t) d t\right]^{1 / p} \\
& =\frac{1}{N}\left[\int_{0}^{\infty}\left(\sum_{k=1}^{N}\left(\sum_{j=1}^{k} 2^{j}\right) \chi_{\left[\left|A_{k+1}\right|,\left|A_{k}\right|\right)}\right)^{p} w(t) d t\right]^{1 / p} \\
& =\frac{1}{N}\left(\sum_{k=1}^{N}\left(\sum_{j=1}^{k} 2^{j}\right)^{p} \int_{\left|A_{k+1}\right|}^{\left|A_{k}\right|} w(t) d t\right)^{1 / p} \\
& \geq \frac{1}{N}\left(\sum_{k=1}^{N}\left(2^{k+1}-2\right)^{p}\left(2^{-k p}-2^{-(k+1) p}\right)\right)^{1 / p} \\
& \geq C_{p} \frac{1}{N} N^{1 / p} \underset{N \rightarrow \infty}{\longrightarrow} \infty \cdot \square
\end{aligned}
$$

Remark 3.3. The above result is not true for general measures. In fact, if $d u=$ $\delta_{0}$, the Dirac delta at the origin, then $\|f\|_{\Lambda_{u}^{p}(w)}=|f(0)|\left(\int_{0}^{1} w(t) d t\right)^{1 / p}$. Hence, $\Lambda_{u}^{p}(w) \approx \mathbb{R}$, for all $p>0$.

\section{INDICES}

Given $\Lambda^{p}(w)$ we define the following invariant index:

Definition 4.1. Given $k=1,2, \cdots$ set

$$
D_{k}=\sup _{t>0} \frac{W\left(2^{k} t\right)}{W(t)}
$$

and

$$
p(w)=\inf \left\{p>0 ; \text { for some } C>0, D_{k} \leq C 2^{k p}, k=1,2, \cdots\right\}
$$

We now prove the fundamental property of $p(w)$ :

Theorem 4.2.

$$
D_{k}^{1 / k} \underset{k \rightarrow \infty}{\longrightarrow} 2^{p(w)}
$$

Proof. Set $a_{k}=\log D_{k}$. Then $a_{j+k} \leq a_{j}+a_{k}$ and therefore there exists $l \geq 0$ such that $a_{k} / k \longrightarrow l$, as $k \rightarrow \infty$ (this is an easy exercise, see for example [GuR]). Thus, $D_{k}^{1 / k} \underset{k \rightarrow \infty}{\longrightarrow} D=\mathrm{e}^{l}$. So, we need to show that $D=2^{p(w)}$. If $p>p(w)$, then $D_{k} \leq C 2^{k p}$ and hence $D \leq 2^{p}$; i.e., $D \leq 2^{p(w)}$. Conversely, if $D<2^{p(w)}$, choose $0<p<p(w)$ such that $D<2^{p}<2^{p(w)}$. Let $k_{0} \in \mathbb{N}$ such that $D_{k}^{1 / k} \leq 2^{p}$, if $k \geq k_{0}$. Let $C=\max \left\{1, D_{k} / 2^{k p}: k=1, \cdots, k_{0}-1\right\}$. Then $D_{k} \leq C 2^{k p}, k \in \mathbb{N}$, and we reach the contradiction $p(w) \leq p<p(w)$. 
Remarks 4.3. (i) As a consequence of this result, it turns out that if two weights $w_{0}, w_{1}$ satisfy $W_{0} \approx W_{1}$, then $p\left(w_{0}\right)=p\left(w_{1}\right)$.

(ii) It is not true, in general, that $D_{k}=D^{k}$. For this, it suffices to consider the weight $w(t)=\chi_{(0,1)}(t)+2 \chi_{[1, \infty)}(t)$. Here, $D_{k}=2^{k+1}-1$.

(iii) The definition of $p(w)$ is closely related to the condition on the weights $R_{p}$ in [Ne] (notice that $R_{1}=B_{1, \infty}$ ).

The following result will be needed later. Recall that $B_{1, \infty} \subset \bigcap_{p>1} B_{p}$.

Lemma 4.4. There exists $w \in \bigcap_{p>1} B_{p} \backslash B_{1, \infty}$.

Proof. We choose a decreasing sequence $\left\{p_{k}\right\}_{k}$, with $\lim _{k} p_{k}=1\left(p_{k} \neq 1, k=\right.$ $1,2, \cdots)$. Consider also $\left\{a_{k}\right\}_{k}, a_{k}>0$ and $\sum_{k} a_{k}=1$. Let us define

$$
w(t)= \begin{cases}\sum_{k=1}^{\infty} a_{k} p_{k} t^{p_{k}-1} & \text { if } 0 \leq t \leq 1 \\ 0 & \text { if } t>1\end{cases}
$$

It is clear that the series converges uniformly on $[0,1]$. Now,

$$
W(t)= \begin{cases}\sum_{k=1}^{\infty} a_{k} t^{p_{k}} & \text { if } 0<t \leq 1 \\ 1 & \text { if } t>1\end{cases}
$$

Thus, $t^{-1} W(t) \longrightarrow 0$, as $t \rightarrow 0$, and therefore $w \notin B_{1, \infty}$. To prove that $w \in B_{p}$, $p>1$, it suffices to show that if $p \neq p_{k}$, there exists $C_{p}>0$ such that condition (1) holds, for $0<r<1$. Now,

$$
\int_{r}^{\infty} \frac{w(t)}{t^{p}} d t=\sum_{k=1}^{\infty} a_{k} p_{k} \int_{r}^{1} t^{p_{k}-1-p} d t=\sum_{k=1}^{\infty} \frac{a_{k} p_{k}}{p_{k}-p}\left(1-r^{p_{k}-p}\right)
$$

and

$$
r^{-p} \int_{0}^{r} w(t) d t=\sum_{k=1}^{\infty} a_{k} r^{p_{k}-p}
$$

Thus, if we let $S_{p}=\sup _{k} p_{k} /\left(p_{k}-p\right)>0, I_{p}=\inf _{k} p_{k} /\left(p_{k}-p\right)<0$ and $D_{p}=$ card $\left\{k: p_{k}>p\right\}$, and choose $k_{0}$ so that $p_{k_{0}}<p$, then with $C_{p}=D_{p} S_{p} / a_{k_{0}}-I_{p}$, we obtain the result.

Theorem 4.5. (a) If $1 \leq p<\infty$, then $w \in B_{p}$ if and only if $p(w)<p$.

(b) If $w \in B_{1, \infty}$, then $p(w) \leq 1$, and the converse is not true in general.

(c) If $\Lambda^{p(w)}(w)$ is a Banach space, then $p(w)=1$, and the converse is not true in general.

Proof. (a) If $w \in B_{p}$ then (see $[\mathrm{AM}]$ ) there exists $q<p$ so that $w \in B_{q}$. Now

$$
\frac{1}{2^{k q} t^{q}} \int_{t}^{2^{k} t} w(s) d s \leq \int_{t}^{2^{k} t} \frac{w(s)}{s^{q}} d s \leq \frac{C}{t^{q}} \int_{0}^{t} w(s) d s
$$

and hence,

$$
\int_{0}^{2^{k} t} w(s) d s \leq\left(1+C 2^{k q}\right) \int_{0}^{t} w(s) d s
$$


which implies $D_{k} \leq C 2^{k q}$. Conversely, if $D_{k} \leq C 2^{k q}$, with $q<p$, then for $r>0$,

$$
\begin{aligned}
\int_{r}^{\infty} \frac{w(t)}{t^{p}} d t & =\sum_{k=0}^{\infty} \int_{2^{k} r}^{2^{k+1} r} \frac{w(t)}{t^{p}} d t \leq \frac{1}{r^{p}} \sum_{k=0}^{\infty} \frac{1}{2^{k p}} \int_{0}^{2^{k+1} r} w(t) d t \\
& \leq \frac{1}{r^{p}} \sum_{k=0}^{\infty} \frac{D_{k+1}}{2^{k p}} \int_{0}^{r} w(t) d t \leq \frac{2^{q} C}{r^{p}}\left(\sum_{k=0}^{\infty} \frac{1}{2^{k(p-q)}}\right) \int_{0}^{r} w(t) d t
\end{aligned}
$$

(b) This is clear by definition. Now if $w \in \bigcap_{p>1} B_{p} \backslash B_{1, \infty}$, as in Lemma 4.4, then $p(w) \leq 1$ (in fact $p(w)=1$ ).

(c) By Corollary 3.2, we have that $p(w) \geq 1$. If $p(w)>1$, then $w \in B_{p(w)}$ and by (a) we obtain a contradiction. Now, with $w$ as in (b), we show that the converse does not hold.

Remarks 4.6. (i) An equivalent result to part (a) of the previous theorem was proved by Raynaud ([Ra]), using more complicated arguments.

(ii) If $w(t)=t^{\alpha}$, with $-1<\alpha \leq 0$, then $p(w)=1+\alpha$, and so $p(w) \leq 1$ is the best we can say in (b) of the theorem.

(iii) It is easy to show that $D_{k} \leq C 2^{k p}$ is equivalent to saying that $W$ is a $p$-quasi-concave function.

(iv) There are other indices (Simonenko, Matuszewska-Orlicz, etc.) for which it is possible to show the equivalence with $p(w)$. In particular $p(w)=\beta_{W}^{a}=\sigma_{W}^{a}=$ $B_{W}^{a}=Q_{W}^{a}$ (see $[\mathrm{Ma}]$ for the definitions).

\section{REFERENCES}

[AM] M.A. Ariño and B. Muckenhoupt, Maximal functions on classical Lorentz spaces and Hardy's inequality with weights for nonincreasing functions, Trans. Amer. Math. Soc. 320 (1990), 727-735. MR 90k:42034

[BS] C. Bennett and R. Sharpley, Interpolation of operators, Academic Press, New York, 1988. MR 89e: 46001

[CS1] M.J. Carro and J. Soria, Weighted Lorentz spaces and the Hardy operator, J. Funct. Anal. 112 (1993), 480-494. MR 94f:42025

[CS2] _ Boundedness of some integral operators, Canad. J. Math. 45 (1993), 1155-1166. MR 95d: 47064

[CS3] _ The Hardy-Littlewood maximal function and weighted Lorentz spaces, J. London Math. Soc. (to appear).

[GR] J. García-Cuerva and J.L. Rubio de Francia, Weighted norm inequalities and related topics, Math. Stud., vol. 116, North-Holland, Amsterdam, 1985. MR 87d:42023

[GuR] M. de Guzmán and B. Rubio, Problemas, conceptos y métodos del análisis matemático I, Ediciones Pirámide, 1990.

[Hu] R.A. Hunt, On L $(p, q)$ spaces, Enseign. Math. (2) 12 (1966), 249-276. MR 36:6921

[KPS] S.G. Kreı̆n, Ju.I. Petunin, and E.M. Semenov, Interpolation of linear operators, Transl. Math. Monographs, vol. 54, Amer. Math. Soc., Providence, RI, 1982. MR 84j:46013

[Lo] G.G. Lorentz, On the theory of spaces $\Lambda$, Pacific J. Math. 1 (1951), 411-429.

[Ma] L. Maligranda, Indices and interpolation, Dissertationes Math. 234 (1985). MR 87k:46059

[Ne] C.J. Neugebauer, Weighted norm inequalities for averaging operators of monotone functions, Publ. Mat. 35 (1991), 429-447. MR 93m:26042

[Ra] Y. Raynaud, On Lorentz-Sharpley spaces, Israel Math. Conf. Proc. 5 (1992), 207-228. MR 94c:46061 
[Sa] E. Sawyer, Boundedness of classical operators on classical Lorentz spaces, Studia Math. 96 (1990), 145-158. MR 91d:26026

[Sj] P. Sjögren, How to recognize a discrete maximal function, Indiana Univ. Math. J. 37 (1988), 891-898. MR 90f:42020

(M. J. Carro and J. Soria) Departamento Matemàtica Aplicada i Anàlisi, Universidad De Barcelona, 08071 Barcelona, Spain

E-mail address: carro@cerber.mat.ub.es

E-mail address: soria@cerber.mat.ub.es

(A. García del Amo) Departamento Análisis Matemático, Facultad de Ciencias, Matemáticas, Universidad Complutense, 28040 Madrid, Spain

Current address: Departamento Matemática Pura y Aplicada, Facultad de Ciencias, Universidad de Salamanca, 37008 Salamanca, Spain

E-mail address: garciada@mat.ucm.es 